\title{
Angular momentum coupling in molecular Rydberg states
}

\author{
J. A. Dagata*, L. Klasinc and S. P. McGlynn \\ Department of Chemistry, Louisiana State University, Baton Rouge, LA 70803 USA
}

\begin{abstract}
The coupling of electronic and nuclear motions in the rovibronic levels of the lowest Rydberg states of methyl iodide is discussed in terms of a frame transformation between lab- and bodyframe coordinate systems for an s Rydberg electron in the field of an electronically-degenerate, symmetric-top molecular ion core. It is shown that the $r$-dependence of exchange, spin-orbit, spin-rotation, and vibronic interactions, where $r$ is the radial distance of the electron from the ion core, must be considered in order to provide a consistent analysis of the experimental data. Molecular constants, obtained by fitting one- and two-photon absorption spectra, show conclusively that the spin of the Rydberg electron is uncoupled from the molecular frame. Bandshape fitting reveals that the interaction between electronic and vibrational angular momenta leads to complete angular momentum quenching in some vibronic levels, in which it appears that the vibrating core "traps" the Rydberg electron. The magnitudes of the B rotational constants permit extrapolation of channel mixing across ionization thresholds in $\mathrm{CH}_{3} \mathrm{I}$ but not in $\mathrm{HI}$. A theoretical frame for the discussion of these events is sketched.
\end{abstract}

\section{A. INTRODUCTION}

This paper is the latest of a series ${ }^{1-7}$ which attempts to apply multichannel quantum defect theory ${ }^{8-10}$ (MQDT), the frame transformation aspect of it, or both, to polyatomic systems. QDT procedures have been successfully applied to a variety of problems in atoms 9,11 and diatomics ${ }^{12}$, but progress for polyatomics has come very slowly. In this work, we will be concerned with a very limited portion of the VUV spectrum of methyl iodide (i.e., the $6 \mathrm{sR}$, or lowest $s$ Rydberg region), and we will focus on a particular ingredient of MQDT, namely frame transformations 10,11 .

The wavefunction of a Rydberg electron with low angular momentum extends over small-r and large-r regions, where different physical situations prevail ${ }^{8}$. The descriptions of the system in these two regions, in terms of alternative expansions of the total wavefunction, are relatable by a transformation of the orbital and spin coordinates of the Rydberg electron from a molecule-fixed coordinate system, in which the initial photoabsorption event occurs, to a laboratory frame coordinate system, one more convenient for representing the motions of a spatially-extended Rydberg electron. Most discussions of polyatomic Rydberg states have emphasized the large-r character of the electron orbital and neglected small-r, off-diagonal effects which mix different electronic, vibrational and rotational channels.

The purpose of this work is to develop frame transformation theory for a Rydberg electron in the field of an electronically-degenerate, symmetric-top molecular ion core, and to use it to classify rovibronic Rydberg states. The choice of the lowest Rydberg states of $\mathrm{CH}_{3} \mathrm{I}$ is convenient because these states are more or less uncontaminated by valence states and are so isolated from higher Rydberg members that the multichannel aspects of $\ell$-mixing can be neglected. Furthermore, because of the strong internal spin-orbit coupling in the ion core, Jahn-Teller distortions will be negligible. On the other hand, intraconfigurational mixing induced by spin-orbit, exchange, and the nuclear kinetic energy terms will be found to be significant.

Methyl iodide, since the early days of molecular spectroscopy, has been a focus of molecular UV and VUV studies, yet much of its more interesting physics remains to be uncovered. It is hoped that our discussion of rovibronic effects will facilitate continued probings of these Rydberg manifolds. 
It is now appropriate to summarize the observations/deductions about the Rydberg manifold of $\mathrm{CH}_{3} \mathrm{I}$ that speak to MQDT and/or transformation theory. These are:

1. The frame transformation method has been applied ${ }^{1}$ to the rotational band shape analyses of one-5 and two-photon ${ }^{6}$ 6sR absorption spectra of $\mathrm{CH}_{3} \mathrm{I}$. It has been shown that uncoupling of spin angular momentum of the optical electron from the molecular axis was essentially complete. The same result may be deduced ${ }^{2}$ from an empirical correlation of the entire experimental data set.

2. It has been shown that the $6 \mathrm{sR}$ photoabsorption event must be viewed as a two-step process $^{3}$ : In the first, the initial electronic excitation accords with the BornOppenheimer Approximation (BOA), while the second consists of angular uncoupling of the Rydberg electron as it escapes from the rotating molecular core.

3. An MQDT analysis of the high-resolution absorption spectrum of $\mathrm{CH}_{3} \mathrm{I}$ has been performed ${ }^{4}$ for energy regions above and below the first ionization limit. It has been shown that spin-orbit coupling and $\ell$-mixing of Rydberg states built on a doublet ion core account for nearly all major aspects of the spectrum. The ability to extrapolate channel mixing in $\mathrm{CH}_{3} \mathrm{I}$ across threshold from the autoionization region to the discrete region, and the inability to do so in $\mathrm{HI}$, is attributable to the much smaller value of the $B$ rotational constant for $\mathrm{CH}_{3} \mathrm{I}$.

4. Nuclear-electronic coupling was observed $1,5,6,7$ in the $v_{6}$ vibronic bands of $\mathrm{CH}_{3} I$ that are built on the $6 \mathrm{sR}$ origins. This coupling produced ${ }^{7}$ almost complete quenching of angular momentum in some such levels.

5. The vibrating nuclear frame ( $v_{6}$ mode, as discussed above) increases the region of the electron core/optical electron interactions to larger radial distances. It appears ${ }^{7}$ that the vibrating core "traps" the Rydberg electron.

6. A transference of angular momentum between other, almost degenerate vibronic pairs has also been observed ${ }^{7}$. It was shown that the coupling operator was rovibronic, the most important part of it being analogous to the Renner effect in linear molecules. However, because of the importance of Coriolis coupling in the non-linear, symmetric top molecule $\mathrm{CH}_{3} \mathrm{I}$, this vibronic, Renner-like part does possess first-order effects.

7. Valence/Rydberg interactions were observed on both sides of the ionization limit(s) and were interpreted. These interactions, while extensive and solely responsible for the photochemistry associated with Rydberg excitations below the first ionization limit, do not vitiate any of the above conclusions/deductions.

The emphasis of the present work resides in statements $1,2,4,5$ and 6 . Connections to statements 3 and 7 obviously exist, but these connections will not be explored in detail.

\section{B. ROTATIONAL-ELECTRONIC STRUCTURE OF THE 6SR ORIGIN BANDS}

The electronic structure of the 6sR states of methyl iodide was first elucidated by Mulliken $13,14,15$. These states are formed by promotion of an electron from the filled $2 \mathrm{e}$ orbital (essentially a nonbonding iodine AO) to the Rydberg $6 \mathrm{~s}$ (6sR) orbital 16 . Term symbols and energies of the $6 \mathrm{sR}$ states are listed in Table 1. Herzberg ${ }^{17}$ discussed the splittings of this configuration in the $(\Lambda, s)$ and $(\Omega, w)$ electronic coupling limits, where $\Lambda$ and $S$ denote the projections of the total orbital and total spin electronic angular momenta onto the molecular axis; $\Omega$ refers to the same projection of the total (orbital plus spin) electronic angular momentum of the core electrons and $w$ specifies the same projection of the total angular momentum of the Rydberg electron. The characteristic features of the $\mathrm{CH}_{3} \mathrm{I}$ photoabsorption spectrum are:

-.- 6sR configurational splittings are quantitatively described by spin-orbit, spin-spin and exchange interaction energies.

-.- The importance of the static Jahn-Teller effect to vibronic structure depends on the strength of spin-orbit coupling and varies inversely with the splitting of core degeneracy. First-urder vibronic distortions, therefore, are minimal, and a $C_{3}$ molecular symmetry group is appropriate for classification of the rovibronic levels.

--- The rotational envelopes of the $6 \mathrm{sR}$ origin bands are not much broadened by predissociative Rydberg-valence interactions. Indeed, the half-bandwidths are consistent with the assumption of small differences in the moments of inertia of upper and lower states.

--- The molecular constants for the $6 \mathrm{sR}$ states are given in Table 2. The results of Table 2 differ substantially from those of Herzberg ${ }^{17}$, Mulliken and Teller ${ }^{13}$, and Parker et al. 18 . 
The very different values we report for the rotational constants $A$ and the effective internal angular momentum $\zeta_{\text {eff }}$ arise because the possibility of a spin uncoupling of the Rydberg electron from the molecular axis had not been considered previously.

-- The Born-Oppenheimer Approximation (BOA) is inapplicable to the $6 \mathrm{sR}$ states of the rotating molecule: the spin projection of the $6 \mathrm{sR}$ electron ceases to be a good quantum number, because of the short-range nature of exchange, outside the core. While the energy spacing of electronic origins can be fully understood within the $B O A$ [i.e., as coupling intermediate to the $(\Lambda, S)$ and $\left(\Lambda_{c}, w\right)$ limits], the form of the final-state angular momentum must be explicitly included in order to interpret the rotational fine structure of the $6 \mathrm{sR}$ bands.

\section{FRAME TRANSFORMATION RELATIONS}

A frame transformation relation connects alternative expansions of the full wavefunction constructed in different regions of molecular configuration space. The most familiar example is the intermediate coupling 10,11 lying between the various Hund's cases for a diatomic. The appropriate partitioning of the configuration space of a homonuclear diatomic was depicted schematically in Fig. 1 of Chang and Fano ${ }^{11}$ and is represented by bracketed quantum numbers in Fig. 1 of the present article. The quantum numbers for a symmetric-top are also shown in Fig. 1. An explication of Fig. 1 proceeds as follows:

-.- Global quantum numbers are given on line a). Lines b)-d) represent various local (approximate) solution of the full Hamiltonian. Roelectronic mixing is shown on line b). Additional effects induced by vibronic interactions are depicted on line d). Long-range orbital angular momentum coupling is represented on line c).

-- Rotational analysis of the $6 \mathrm{sR}$ origin bands provides information about the coupling represented on line b) for a pure s electron. Analysis of the $6 \mathrm{sR}$ vibronic bands leads to information about the coupling indicated on lines $b$ ) and $d$ ), namely the effect of nuclear vibrations on the escape of the Rydberg electron from the region $r<r$. Because $r_{1}$ extends beyond the core, rotational constants for the vibronic transitions may differ from those of the origin band because of the nature of their angular momentum coupling.

-- Beyond the $6 \mathrm{sR}$ region (i.e., for $E>58000 \mathrm{~cm}^{-1}$ ), the spectrum is complicated by Rydberg-Rydberg and Rydberg-valence interactions. The regular Rydberg progressions beginning about $74000 \mathrm{~cm}^{-1}$ can now be modeled by MQDT parametrization of the long-range orbital angular momentum coupling. The range of this coupling is indicated by the boundary $r_{2}$.

-- Between $58000 \mathrm{~cm}^{-1}$ and $75000 \mathrm{~cm}^{-1}$, Rydberg-valence interactions exert substantial effects on Rydberg series members. The generalized MQDT approach of Guisti 19 suggests that the underlying valence states of the molecule may be probed by this technique.

The frame transformation is diagonal in the rovibronic quantum number ${ }^{20} \mathrm{G}$ and the total angular momentum $J$ of the system. Various local quantum numbers of the region $A$ (region B) descriptions of Fig. 1, which describe the small-r (large-r) behavior of the molecular complex (fragments), are determined by separation of the full Hamiltonian (see Appendix). The Chang-Fano formulation of the frame transformation matrix for a homonuclear diatomic ${ }^{11}$ is readily generalized to an $s$ Rydberg of a symmetric-top molecule by introducing restrictions on the values of the symmetric-top quantum numbers $K$ and $K$, where $K(K)$ represents the projection of the total angular momentum onto the moleccular axis for the ion core (molecular complex), which can enter into the vector coupling coefficients. These additional selection rules are important to a correct accounting of the uncoupling of $6 \mathrm{sR}$ angular momentum from the molecular axis in the presence of first-order Coriolis coupling: It is necessary to distinguish the direction of rotation about the molecular axis for the sum of the electronic and nuclear angular momentum vectors. For example, for the case of an s-electron:

$$
\mathrm{K}_{\mathrm{c}}=\mathrm{K} \pm 1 / 2= \pm \Omega \pm \mathrm{k} \pm 1 / 2
$$

This "bookkeeping" is conveniently handled by the quantum number $G$.

According to line b) of Fig. 1, the spin projection of the $6 \mathrm{sR}$ electron on the molecular axis is only meaningful when $r<r$. In this region, an intermediate coupling description of the electronic angular momentum is required since spin-orbit coupling and exchange terms are both large. The local rotational-electronic quantum numbers for region $A$ are given by the sets 
TABLE 1. Symmetry species, energies and quantum defects for the ground state and electronic states of the $6 \mathrm{sa}_{1}$ Rydberg configuration of $\mathrm{CH}_{3} \mathrm{I}$.

\begin{tabular}{|c|c|c|c|c|c|}
\hline \multirow{2}{*}{$\begin{array}{c}\text { STATE } \\
\#\end{array}$} & \multicolumn{3}{|c|}{ SYMETRY SPECIES } & \multirow{2}{*}{$\begin{array}{l}\text { ENERGY } \\
\left(\mathrm{cm}^{-1}\right)\end{array}$} & \multirow{2}{*}{$\begin{array}{c}\text { QUANTUM DEFECT } \\
\mu(I P)^{c}\end{array}$} \\
\hline & $c_{\infty \mathrm{v}}$ & $c_{3 v}^{a}$ & $c_{3 v}^{b}$ & & \\
\hline $\begin{array}{l}0 \\
1 \\
2 \\
3 \\
4\end{array}$ & $\begin{array}{l}{ }^{1} \Sigma^{+}\left(0^{+}\right) \\
{ }^{3} \Pi(2) \\
{ }^{3} \Pi(1) \\
{ }^{3} \Pi(0 \pm) \\
{ }^{1} \Pi(1)\end{array}$ & $\begin{array}{c}A_{1} \\
E \\
E \\
A_{1}, A_{2} \\
E\end{array}$ & $\begin{array}{l}6 s ;{ }^{2} E_{3 / 2} \\
6 s ;{ }^{2} E_{3 / 2} \\
6 s ;{ }^{2} E_{1 / 2} \\
6 s ;{ }^{2} E_{1 / 2}\end{array}$ & $\begin{array}{r}0 \\
49201 \\
49715 \\
54030 \\
54640\end{array}$ & $\begin{array}{c}- \\
4.010\left(I_{1}\right) \\
3.992\left(I_{1}\right) \\
4.017\left(I_{2}\right) \\
3.997\left(I_{2}\right)\end{array}$ \\
\hline
\end{tabular}

a) In a $(\Lambda, S)$ coupling limit.

b) In a $(\Omega, w)$ coupling limit.

c) Relative to the ${ }^{2} \mathrm{E}_{3 / 2}\left(\mathrm{I}_{1}\right)$ or ${ }^{2} \mathrm{E}_{1 / 2}\left(\mathrm{I}_{2}\right)$ ion core limits of $\mathrm{CH}_{3} \mathrm{I}^{+}$.

TABLE 2. Molecular constants $4,5,6$ of the $6 \mathrm{sR}$ electronic levels of $\mathrm{CH}_{3} \mathrm{I}$.

\begin{tabular}{lccc}
\hline \multirow{2}{*}{ STATE } & \multicolumn{2}{c}{$\begin{array}{c}\text { ROTATIONAL CONSTANTS } \\
\left(\mathrm{cm}^{-1}\right)\end{array}$} & $\begin{array}{c}\text { INTERNAL ANGULAR } \\
\text { MOMENTUM }\end{array}$ \\
\cline { 2 - 3 } & A & B & $\zeta_{\text {eff }}$ \\
\hline 0 & 5.119 & 0.250 & 0 \\
1 & 4.343 & 0.245 & 1.40 \\
2 & 4.446 & 0.245 & 1.30 \\
3 & 4.906 & 0.245 & 0.70 \\
4 & 5.008 & 0.240 & 0.65 \\
\hline
\end{tabular}

a) All excited-state values are correct to about $5 \%$. The ground state constants are taken from Ref. 17.

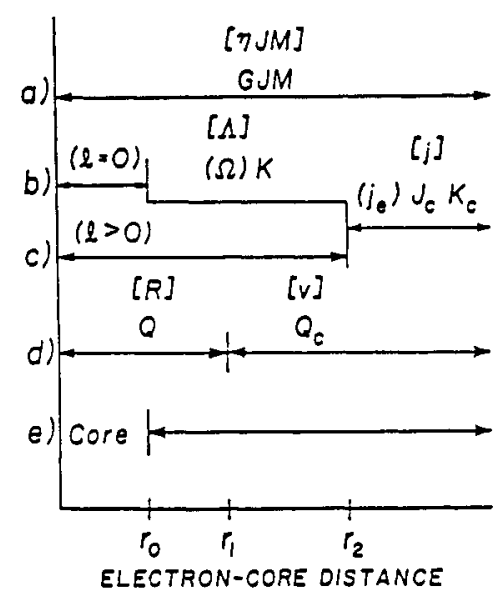

region $A$

Figure 1. Diagram showing quantum numbers for a symmetric top molecule for different ranges of the electron-core distance $r$. Quantum numbers in brackets relate to a homopolar diatomic and are those given in Fig. 1 of Ref. 11. See text for definitions of symbols and different coupling regions. 


$$
\{\alpha\}=\{\Omega(\Lambda, S), K\} ;\{\bar{\alpha}\}=\left\{\Omega\left(\Omega_{c}, \omega\right), K\right\}
$$

in which the idealized electronic angular momentum coupling schemes are given as well. Spin-orbit coupling is a purely electronic component of the overall frame transformation matrix elements:

$$
\langle\alpha \mid \bar{\alpha}\rangle=\left\langle\Lambda, s \mid \Omega_{c}, \omega\right\rangle
$$

The region $B$ description, on the other hand, corresponds to a situation in which the orbital and spin coordinates of the Rydberg electron refer to a lab frame system. The quantum numbers for a $6 \mathrm{sR}$ state in this representation are

$$
\{i\}=\left\{s, \Omega_{c}, K_{c}, J_{c}\right\}
$$

where $s$ is the spin of the Rydberg electron and $\Omega_{c}, K_{c}$ and $J_{c}$ are core variables.

The overall frame transformation connecting regions $A$ and $B$, which is denoted $\langle i \mid \alpha\rangle$, cannot be constructed by simply letting $k \rightarrow K$ ( $i . e$. , independently of the values of $\Omega$ and $\Omega_{\mathrm{J}}$ ). First-order Coriolis coupling is significant in both the molecular complex and the ion core and this coupling spoils the usefulness of the individual rotational and electronic quantum numbers $K, \Omega$ and $\Omega$ as the system evolves from region $A$ to region $B$. For region $A$, the projection of the total angular momentum on the molecular axis is

$$
K=k+\zeta_{e f f}
$$

where $\zeta_{\text {eff }}$, in the absence of vibronic coupling, is

$$
\xi_{\text {eff }} \leqslant \Omega\left|(L+S)_{z}^{2}\right| \Omega>
$$

because $\overline{(\mathrm{L}+\mathrm{S})^{2}}=\overline{(\mathrm{L}+\mathrm{S})^{2}}=0$. The projection of the total angular momentum on the molecular axis of the holecular ion core in region $\mathrm{B}$ is given by a similar expression, namely

$$
K_{c}=k+\xi_{\text {eff }}^{+}
$$

and is related to the $K$ levels of the molecular complex by Eq. 1 . The quantity $\xi^{+}$eff is given approximately by $\Omega_{c}=3 / 2,1 / 2$.

Hougen ${ }^{20}$ noted the invariance of the total wavefunction of a symmetric-top molecule to permutations of identical nuclei and used it to classify their rovibronic levels. Thus, the quantum number $21 \mathrm{G}$ is a bookkeeping device for the direction of the total electronic $\left(G_{e}\right)$, vibrational $\left(G_{v}\right)$, and rotational (k) angular momenta about the symmetry axis:

$$
G=G_{e}+G_{v}-k
$$

$G$ has the advantage that it is conserved during the initial, purely electronic stage of photoabsorption and during the uncoupling of the molecular complex into the final states. Again following Hougen ${ }^{20}$, it is convenient to specify degenerate pairs of roelectronic levels by

$$
\begin{aligned}
& G_{a}= \pm\left(\left|G_{e}\right|-k\right) \\
& G_{b}= \pm\left(\left|G_{e}\right|+k\right) .
\end{aligned}
$$

The selection rules for $G$ are represented here by $\langle G\rangle$, where

$$
\langle G\rangle=1 \text {, if } G_{a}=G_{a}^{+} \text {or } G_{b}=G_{b}{ }^{+} ;\langle G\rangle=0 \text {, otherwise }
$$


and where the symbols $\mathrm{G}^{+}$and $\mathrm{G}^{+}$label the rovibronic levels of the freely rotating molecular ion core. The overall frame transformation for the $6 \mathrm{sR}$ states then becomes

$$
\langle i \mid \alpha\rangle^{G J}=\left[\left(2 J_{c}+1\right) /(2 J+1)\right]^{1 / 2}\left(1 / 2 \Omega_{c} J_{c} K_{c} \mid 1 / 2 J_{c} J K\right)\langle i \mid \alpha\rangle\langle G\rangle
$$

The part of the spin uncoupling operator that is of interest here induces mixing, as in a diatomic molecule, by "end over end" rotations. Thus, the off-diagonal matrix elements in the region $A$ representation are

$$
\left\langle\chi^{\alpha}\left|v_{J S}\right| x^{\alpha^{\prime}}\right\rangle=(1 / 2)\left\langle x^{\alpha}\left|J_{+} S_{-}+J_{-} S_{+}\right| x^{\alpha^{\prime}}\right\rangle
$$

where $\chi^{\alpha}$ is a function of all electronic and nuclear coordinates except for the radial distance $r$ of the Rydberg electron ${ }^{11}$.

Resolution of the $\mathrm{J} \cdot \mathrm{S}$ operator in $\mathrm{C}_{3 \mathrm{~V}}$ reveals ${ }^{22}$ that the species $\left(\mathrm{J}_{ \pm}\right) \square\left(\mathrm{S}_{ \pm}\right)$possess $\mathrm{E}$ symmetry. This leads to the following selection rules for $\Sigma$, the projection of the total spin on the molecular axis, and $K$ :

$$
\Delta \Sigma= \pm 1 ; \Delta K= \pm 1
$$

Setting $\mathrm{K}=\mathrm{k}+\xi_{\text {eff }}$, as before, the selection rules may be rewritten as either

$$
\Delta \Omega= \pm 1 \text { and } \Delta k=0
$$

where no change occurs in the nuclear rotational quantum number $k$ or as the alternate possibility

$$
\Delta \Omega=0 \text { and } \Delta k= \pm 1 \text {, }
$$

where the rotational quantum number changes by unity. Note that Eq. 14 is a familiar result in diatomic spectroscopy but that Eq. 15 has no such counterpart because, for a diatomic, $K \equiv \Omega$.

\section{CLASSIFICATION OF ROVIBRONIC LEVELS}

A set of correlation diagrams, one for state 2 and one for state 3 , is given in Figs. $2 a, b$. These correlation diagrams are vested in the discussion of the previous section and will be discussed in some detail. In the discussion of Figs. 2a,b, we will distinguish the values of $k$ in regions $A$ and $B$ by $k^{\prime}$ and $k^{\prime \prime}$, respectively. We impose this distinction because the possibility of a slight zero-point shift of the levels, attributable to spin contributions, should occur.

The empirical parameters of bandshape fittings ${ }^{1}$ are the rotational constants, $A$ and $B$, and the internal angular momentum, $\zeta$. These parameters, for a given rotational envelope, are obtained from the oscilfator strength distribution and the energy spacing of the sub-bands. The sub-band spacing is given by the difference between $2 A \xi$ for for the upper and lower states of the rotational term energy. Since only the product $A \zeta$ eff can be determined from a single band, care must be exercised in the assignment of individual values to $A$ and $\zeta$ ff. Experience, however, shows that the calculated spectrum is extremely sensitive to small parameter variations in certain neighborhoods of $\zeta$ and $A$ whereas, in other neighborhoods, there is much insensitivity to such variations. insensitive region occurs for $\zeta_{\text {eff }}=1$ and $A^{\prime \prime} \sim A^{\prime}$, where $A^{\prime \prime}$ and $A^{\prime}$ are the upper and lower state constants.

Rovibronic core levels in the region B representation are associated with half integer angular momenta. Since these levels correlate with integer-valued $K$ levels in the region A representation, as shown in Figs. $2 a, b$, consistency with conservation of $G$ must be maintained. The following comments are pertinent to the construction of Fig. 2 . 

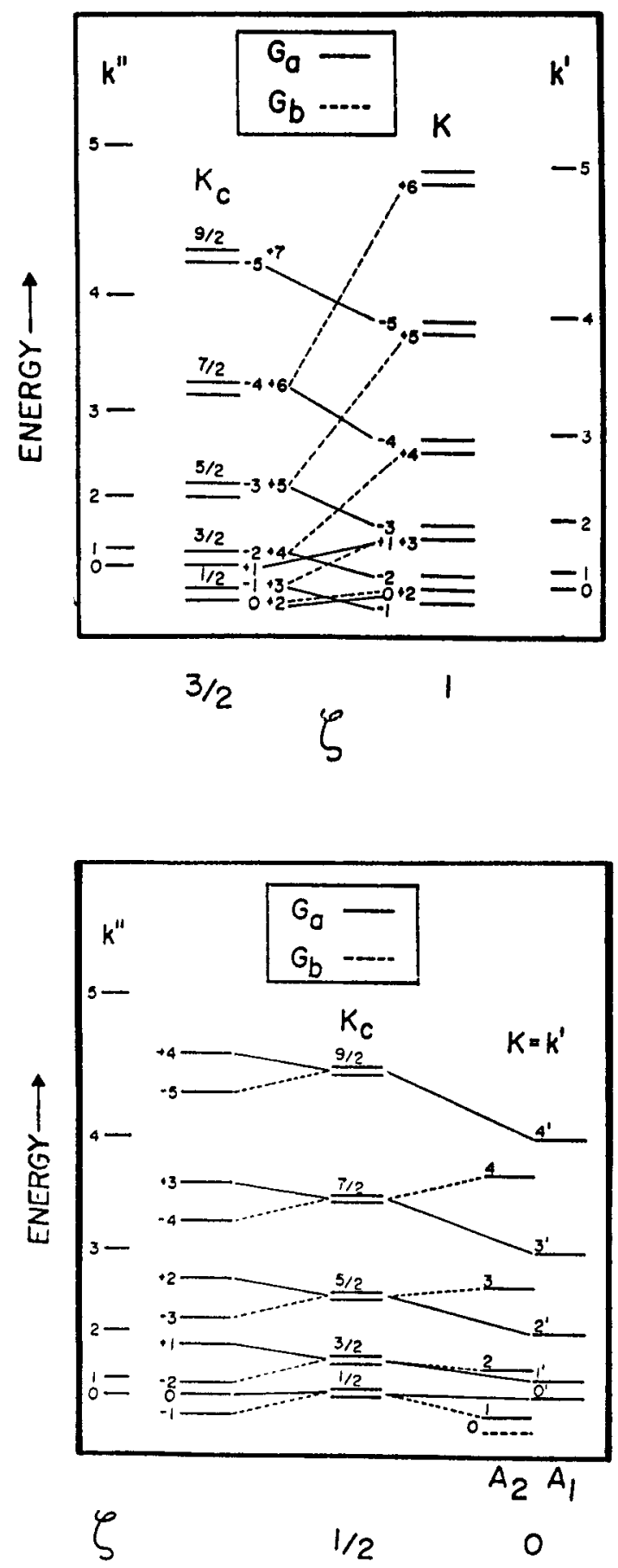

Figure 2. Correlation diagrams for the roelectronic levels of a symmetric top molecule in which both Coriolis and spin uncoupling are important.

a) Diagram for state 2 of the $6 \mathrm{sR}$ configuration of $\mathrm{CH}_{3} \mathrm{I}$. $\mathrm{k}^{\prime}$ and $\mathrm{k}^{\prime \prime}$ are quantum numbers for pure nuclear rotation about the molecular axis in regions $A$ and $B$, respectively. $K$ and $K$ are projections of the total angular momentum on the molecular axis when the spin of the Rydberg electron is alternately referred to the body frame and lab frame, respectively; and $G$ and $G$ are rovibronic quantum numbers for classifying levels of the symmetric tôp. The $\mathrm{K}$ quantum number has been omitted for reasons of clarity; the correlation lines indicating Coriolis splitting of the $k^{\prime}$ and $k^{\prime \prime}$ levels have also been left out. The energy level spacings are roughly to scale.

b) Uncoupling diagram for the $A_{1}$ and $A_{2}$ components of state 3 of the $6 \mathrm{sR}$ configuration. 
--- $k^{\prime}$ and $k^{\prime \prime}$ are the (zero-order) quantum numbers for nuclear rotation in regions $A$ and $B$.

-.- The $G_{a}$ and $G_{h}$ quantum numbers occur in $F i g .2 a$ in the region between $3 / 2<\zeta<1$; in Fig. $2 b$, they occur to the left of the levels for which $\zeta>1 / 2$.

-- Selection rules pertaining to the spin-uncoupling operator were used in making the correlations.

-.- The full spin degeneracy of the region B levels (i.e., Kramer's degeneracy) is not shown.

--- Individual $\mathrm{J}$ levels are not shown because they do not influence the classification scheme.

--- The $\mathrm{K}$ quantum numbers for the individual levels of the region $\mathrm{A}$ description and the doubled nature of the $\mathrm{k}$ levels have been omitted for clarity.

-- Fig. 2a was constructed for state 2 but is equally pertinent to state 1 with slight alternations of the $K$ level spacing. Indeed, since bandshape calculations show that $\xi_{\text {eff }} \sim 3 / 2$ for both states, a separate diagram is superfluous.

-- Mixing of different electronic states $\Omega=2,1,0$, and 1 is not shown. This is equivalent to setting $\langle i \mid \alpha\rangle=1$ in Eq. 11 .

If the spin of the Rydberg electron remained coupled to the molecular axis, as was previously assumed, the mixing of singlet and triplet wavefunctions by the spin-orbit operator would be essentially complete and would yield

$$
\begin{aligned}
& \chi_{2}(\Omega=1)=2^{-\frac{1}{2}}\left[\Phi\left({ }^{3} \Pi_{1}\right)+\Phi\left({ }^{1} \Pi_{1}\right)\right] \\
& \chi_{4}(\Omega=1)=2^{-\frac{1}{2}}\left[\Phi\left({ }^{3} \Pi_{1}\right)-\Phi\left({ }^{1} \Pi_{1}\right)\right]
\end{aligned}
$$

Such complete mixing implies that both states should possess identical rotational envelopes. Since the observed bandshapes are very different in both the one- and twophoton spectra, it is quite clear that the rotational structure of the $6 \mathrm{~s} R$ states of $\mathrm{CH}_{3}$ ! cannot vest solely in a $B O A$ approach.

\section{E. DISCUSSION}

The method of frame transformations ${ }^{10}$ has been applied here to spin uncoupling in Rydberg states of a symmetric-top molecule. The utility of this approach is amply justified by the bandshape simulations of one- and two-photon spectra given by Dagata and McGlynn ${ }^{-5}$. Indeed, in retrospect, it is surprising that BOA breakdown for these isolated $s$ Rydbergs should have escaped attention for so long. It turns out that the effects of spin uncoupling are rather subtle in the one-photon absorption spectra, seeming to confer properties associable with modified vibrations. This latter statement is a consequence of the fact that region $A$ extends to $r>r_{0}$ when vibrations are excited. Since the analyses of Mulliken and Teller ${ }^{13}$, and Herzbergli were limited to the extraction of rotational constants from the sub-band spacing of vibrationally degenerate vibronic levels (i.e., the so-called widely-spaced bands), and since these data were not inconsistent with $B O A$, it was believed that the $6 \mathrm{sR}$ states of $\mathrm{CH}_{3} \mathrm{I}$ were well understood. It was not until twophoton resonant MPI studies ${ }^{18}$ became available that inconsistency was exposed $5,6,7$.

An important connection exists between the application of rotational frame transformations to the 6 sR states ${ }^{1-7}$ and the recent application ${ }^{5}$ of full MQDT procedures to the high-lying Rydberg levels in the "discrete" and autoionizing regions near the first ionization threshold of $\mathrm{CH}_{3} \mathrm{I}$. Unlike the electronically-similar diatomic HI, rotational-electronic mixing is suppressed by the smallness of the $B$ rotational constant of methyl iodide. Furthermore, Coriolis coupling effectively superposes all the symmetric-top rotational sub-bands at or near the band origin. It is then easy to associate band maxima at very high $n$ with a unique electronic origin. Consequently, it was possible to unravel major portions of the discrete and autoionizing regions of $\mathrm{CH}_{3} \mathrm{I}$ using ab initio MQDT parameters ${ }^{23}$ for HI. Similar transference across $I_{1}$, however, was not feasible in HI itself!

\section{Acknowledgements}

The authors wish to thank Prof. A. R. P. Rau (LSU) for his many helpful suggestions. This research was sponsored by the U.S. Department of Energy. 


\section{APPENDIX}

The QDT approach emphasizes the radial motion of the Rydberg electron outside the core region, $r>r_{2}$. The fragmentation channels are specified by a set of quantum numbers \{i\}. The net effect of the short-range electron-core interactions for a given eigenchannel $\alpha$ of the molecular complex outside the core boundary is represented by a quantum defect parameter, $\mu_{\alpha}$. If the number of fragmentation channels $n_{i}$ is greater than one, then the frame transformation relation $\langle i \mid \alpha\rangle$ must be constructed. ${ }^{i}$

In this Appendix, we obtain the relevant short-range interactions by writing the full Hamiltonian

$$
\mathrm{H}=\mathrm{H}_{\mathrm{O}}+\mathrm{V}
$$

in terms of a zero-order Hamiltonian of the form

$$
\mathrm{H}_{0}=\mathrm{H}_{\mathrm{c}}+\mathrm{h}(\mathrm{r})=\left[\left(\mathrm{T}_{\mathrm{N}}^{+}+\mathrm{T}_{\mathrm{e}}^{+}+\mathrm{V}^{+}\right)_{\text {core }}+\mathrm{h}(\hat{\mathrm{r}})\right]+\mathrm{h}(\mathrm{r})
$$

where the terms $(\cdots)$ ) contain nuclear and electronic potential and kinetic energy operators of the $n-1$ efectron molecular ion core, $h(\hat{x})$ is the angular operator of the Rydberg electron and

$$
h(r)=-\left(\frac{1}{2}\right) d^{2} / d r^{2}+\ell(\ell+1) / 2 r^{2}+v_{S C F}
$$

is the effective one-electron Hamiltonian of the Rydberg electron. Assuming that the nuclei are fixed in their equilibrium positions, and that vibronic coupling can be explicitly excluded, the rotational kinetic energy operator appearing in $\mathrm{T}_{\mathrm{N}}^{+}$is given by

$$
\mathrm{T}_{\text {rot }}^{+}=\frac{1}{2} \Sigma_{i} \mu_{i i}^{e}\left[J_{c}-P_{c}-(L+S)_{c}\right]_{i}^{2}
$$

where $\mu_{i j}^{e}$ is the equilibrium moment of inertia tensor of the core and $J_{C}, p_{C}$ and $(L+S) C$ are the total, vibrational and electronic (orbital plus spin) angular mómentum operators of the freely rotating core referred to a molecule-fixed coordinate system (i.e., $i=$ $x, y, z)$. Crossterms between electronic and nuclear motions (e.g., Coriolis and vibronic interactions) are not small in $\mathrm{CH}_{3} \mathrm{I}$ and these must be considered explicitly. A similar expression can be written for the kinetic energy operator $T_{N}$ in the region $A$ description in terms of electronic, vibrational and rotational operators as well. Finally, $\mathrm{T}_{N} \rightarrow \mathrm{T}_{N}^{+}$ as the electron-ion core distance increases.

The separation of $H$ into $h(r)$ and $H_{c}$ is exact for infinitely large separations of the fragments. For finite distances, $V$ is given by a sum of electron-electron interaction operators, $V_{e e}$, and electron-nuclear coupling operators, $V_{e N}$. The range of these terms serves to define an effective core boundary, $r_{2}$. Thus

$$
\mathrm{V}_{\mathrm{SCF}}=-1 / \mathrm{r} ; \mathrm{V}=0 ; \text { for } r>r_{2}
$$

The following terms appear in $\mathrm{V}$ :

--- The exchange operator between the Rydberg electron and the $(n-1)$ core electrons, $\mathrm{V}$ ex;

--- Spin-orbit coupling operators, $\mathrm{V}_{\mathrm{so}}$, that are not included in $\mathrm{H}_{\mathrm{c}}$;

-- Spin-spin operators, $V_{s s}$, that are not included in $H_{c}$;

--- The orbital-rotation operator, $V_{\mathrm{JL}}$, which is responsible for $\ell$-uncoupling;

-.- The spin-rotation operator, $\mathrm{V}_{\mathrm{JS}}$; and

-- The dynamic vibronic interaction operator, $\mathrm{V}_{\mathrm{pL}}$.

The first three terms

$$
\mathrm{v}_{\text {ee }}=\mathrm{v}_{\text {ex }}+\mathrm{v}_{\text {so }}+\mathrm{v}_{\text {ss }}
$$

mix only electronic quantum numbers while the last three,

$$
\mathrm{v}_{\mathrm{eN}}=\mathrm{v}_{\mathrm{JL}}+\mathrm{v}_{\mathrm{JS}}+\mathrm{v}_{\mathrm{pL}}
$$

couple electronic and nuclear motions. Note that Eqn. A7 for a pure $s$ Rydberg state in the absence of degenerate vibrations reduces to

$$
\mathrm{V}_{\mathrm{eN}} \sim \mathrm{v}_{\mathrm{JS}}
$$




\section{REFERENCES}

1. J. A. Dagata and S. P. McGlynn, Int. J. Quantum Chem., S19, 323 (1986).

2. J. A. Dagata, M. A. Scott, W. S. Felps and S. P. McGlynn, "Understanding Molecular Properties," ed. A. E. Hansen and J. P. Dahl, D. Reidel Publishing Co., Dordrecht, 1987 , p. $277-296$.

3. J. A. Dagata and S. P. McGlynn, J. Mol. Struct. (Theochem), 135, 329 (1986).

4. J. A. Dagata. M. A. Scott and S. P. McGlynn, J. Chem. Phys., 88,9 (1988).

5. J. A. Dagata, W. S. Felps and S. P. McGlynn, J. Chem. Phys., 85, 2483 (1986).

6. J. A. Dagata, M. A. Scott and S. P. McGlynn, J. Chem. Phys., $\underline{85}, 5401$ (1986).

7. J. A. Dagata, I. Klasinc and S. P. McGlynn, "Studies in Physical and Theoretical Chemistry, MATH/CHEM/COMP 1988," ed. R. C. Lacher, Elsevier, Amsterdam, 1989, in press.

8. M. J. Seaton, Rep. Prog. Phys. 46, 167 (1983).

9. M. J. Seaton, Proc. Phys. Soc. London 88, 801 (1966); 88, 815 (1966).

10. U. Fano, Phys. Rev. A 2, 353 (1970).

11. E. S. Chang and U. Fano, Phys. Rev. A 6,173 (1972).

12. C. H. Greene and Ch. Jungen, Adv. At. Mol. Phys., 21, 51 (1985).

13. R. S. Mulliken and E. Teller, Phys. Rev. 61, 283 (1942).

14. R. S. Mulliken, Phys. Rev. 57, 500 (1940).

15. R. S. Mulliken, Phys. Rev. $\frac{61}{2}, 277$ (1942).

16. The designation $6 \mathrm{sR}$ refers variously to the $6 \mathrm{sa}_{1}$ orbital, to the configuration $\ldots 1 e^{4} a_{1} 22 e^{3} 6 s a_{1}$, to the states 1-4 derived from this configuration, and to transitions from the ground electronic state to states 1-4.

17. G. Herzberg, "Molecular Spectra and Structure. III. Electronic Spectra and Electronic Structure of Polyatomic Molecules," Van Nostrand and Reinhold Co., New York 1966.

18. D. H. Parker, R. Pandolfi, P. R. Stannard and M. A. El-Sayed, Chem. Phys. 45, 27 (1979).

19. A. Guisti, J. Phys. B 13,3867 (1980).

20. J. T. Hougen, J. Chem. Phys. 37,1433 (1962).

21. The quantum number $G$ is defined as follows: the total molecular wavefunction is invariant to operations of the molecular symmetry (MS) group and, in particular, to permutation of equivalent nuclei. In the $C_{3 \mathrm{v}}$ MS group, permutation can be representen

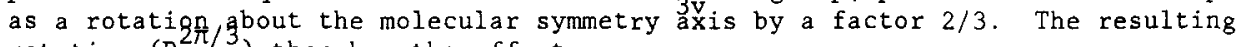
rotation $\left(R_{z}^{2 \pi / 3}\right)$ then has the effect

$$
R_{z}^{2 \pi / 3} \Psi_{J M}=e^{-2 \pi i G / 3} \Psi_{J M}
$$

where $G$ is Hougen's quantum number. The MS group is discussed in detail in Ref. 22.

22. P. R. Bunker, "Molecular Symmetry and Spectroscopy," Academic Press, New York 1979.

23. H. Lefebvre-Brion, A. Guisti-Suzor and G. Raseev, J. Chem. Phys. 83, 1557 (1985). 